\title{
Oromucosal Powder in Pouch Dosage
}

\section{Form}

National Cancer Institute

\section{Source}

National Cancer Institute. Oromucosal Powder in Pouch Dosage Form. NCI Thesaurus.

Code C149739.

Medicinal product consisting of an oromucosal powder presented in a pouch. 